\title{
Influence of Voice Focus on Oral-Nasal Balance in Speakers of Brazilian Portuguese
}

\author{
Gillian de Boer $^{\mathrm{a}}$ Viviane Cristina de Castro Marino ${ }^{\mathrm{b}} \quad$ Larissa Cristina Berti $^{\mathrm{b}}$ \\ Eliana Maria Gradim Fabron ${ }^{b}$ Tim Bressmann ${ }^{a}$ \\ ${ }^{a}$ Department of Speech-Language Pathology, University of Toronto, Toronto, ON, Canada; ${ }^{b}$ Department of \\ Speech-Language Pathology and Audiology, Universidade Estadual Paulista "Júlio de Mesquita Filho", Marília, Brazil
}

\section{Keywords}

Voice focus · Oral-nasal balance · Hypernasality · Brazilian Portuguese speakers · Nasalance

\begin{abstract}
Objectives: This study investigates whether a change in speaking voice focus affects the oral-nasal balance. The investigation was undertaken with different phonetic materials in speakers of Brazilian Portuguese, which features phonological and phonetic vowel nasalization. Methods: Ten females read oral, balanced oral-nasal, and nasal loaded sentences in their normal voice, and with a backward focus and a forward focus. Nasalance scores were collected with a Nasometer 6400. Results: A repeated measures ANOVA of the nasalance scores demonstrated a significant main effect of speaking condition $(F(2,18)=12.87, p<0.001)$. The mean nasalance scores across the stimuli in the backward focus and normal speaking conditions were $36.85 \%$ (SD 16.85) and $40.18 \%$ (SD 18.02), respectively, both significantly lower than the forward focus condition at $45.38 \%$ (SD 18.90). Conclusion: The results demonstrated that speaking focus influences oral-nasal balance in normal speakers. In future research, it should be investigated whether voice focus can also modify oral-nasal balance in hypernasal speakers with cleft palate and other disorders.

๑) 2016 S. Karger AG, Basel
\end{abstract}

\section{Introduction}

Voice focus is a concept from singing pedagogy that has been adapted to voice therapy. The shape and length of the vocal tract determines the timbre of the voice [1]. A shortened vocal tract with a raised larynx, a forward tongue carriage, and a narrowed pharynx results in a bright and juvenile vocal quality (forward focus). A vocal tract lengthened by lowering the larynx, carrying the tongue more posteriorly, and widening the pharynx, results in a dark and throaty voice quality (backward focus) [2]. The lengthening or shortening of the vocal tract changes the frequency distribution in the long-term average spectrum, in particular the second formants (F2) of vowels [3]. In voice therapy, a balanced, central focus is the goal [4].

In a previous study, we investigated whether the speaking focus of the voice influences oral-nasal balance in speech. Oral-nasal balance is regulated by the velopharyngeal mechanism $[5,6]$. Velopharyngeal closure is a task-dynamic process. The degree of velopharyngeal elevation can vary for different tasks such as swallowing, speaking, whistling, or blowing [7-9]. The oral-nasal balance of different vowels varies systematically [10-13]. Different speakers may also vary in their velopharyngeal closure patterns [14].

\section{KARGER}

E-Mail karger@karger.com

www.karger.com/fpl
(C) 2016 S. Karger AG, Basel

1021-7762/16/0683-0152\$39.50/0
Gillian de Boer, MA, MSc

Department of Speech-Language Pathology

University of Toronto, 766-500 University Ave.

Toronto ON M5G 1V7 (Canada)

E-Mail gillian.deboer@mail.utoronto.ca 
If there is velopharyngeal dysfunction related to a structural or neurological disorder, the velopharyngeal mechanism may not close properly, leading to hypernasality. In hypernasal speech, too much air and sound are emitted through the nose, affecting intelligibility and acceptability of speech [15]. Velopharyngeal movement is difficult to influence voluntarily because the velopharyngeal sphincter offers no proprioception [16]. This poses a challenge for behavioral speech therapy in patients with hypernasality. As a result, speech-language pathologists often refer patients with more than mild hypernasality for further surgical management with a pharyngeal flap surgery or for prosthodontic management with a speech bulb or palatal lift prosthesis [17].

Precisely because speakers have little voluntary or proprioceptive control over the state of their velopharyngeal closure mechanism [18], it is of interest whether global vocal tract adjustments such as voice focus can change oral-nasal balance. If consistent changes could be demonstrated, this could potentially open up new possibilities for speech therapy interventions for selected patients with hypernasality. This could be helpful for patients with mild degrees of hypernasality as well as patients learning to use a new pharyngeal flap or speech prosthesis. Based on a study involving computer modeling, Rong and Kuehn [19] speculated that an expanded pharynx and a more anterior tongue position would improve the oral-nasal balance and the perception of hypernasality for the vowel /i/. Conversely, Bressmann et al. [20] described a speaker who reduced her hypernasality and her nasalance scores by adopting a forward focus (raised larynx and narrowed pharynx). The authors speculated that a narrowing of the pharynx may have facilitated velopharyngeal closure.

In a previous study [21], 16 normal female speakers produced 6 test sentences without nasal sounds and 1 test sentence loaded with nasal sounds. The speakers produced the stimuli with their normal voice and with a backward focus and a forward focus. Audio-recordings and nasometry measurements [21] were made. Based on a perceptual evaluation, which was corroborated with long-term average spectra, 9 of the participants were able to complete the task successfully. A repeated measures ANOVA demonstrated that nasalance scores were influenced by the stimulus type (oral vs. nasal stimuli) which was expected. A follow-up ANOVA found a condition effect for the nasal stimulus only, showing that nasalance scores of the backward focus were lower and those of the forward focus were higher than in the normal condition. This indicated that the selection of test sentences was not ideal because the effect of the voice focus appeared to be

Influence of Voice Focus on Oral-Nasa Balance most pronounced on the stimulus including nasal speech sounds. It could be argued that a speaker opens his or her velopharyngeal sphincter intermittently for a nasal sentence. Therefore, a change in voice focus can affect the distribution of sound between the oral and the nasal cavities during these opening gestures. However, the velopharyngeal sphincter remains closed for the whole duration of an oral stimulus, so a forward or backward voice focus would only affect transpalatal resonances.

The goal of the present study was to expand on the previous research [21]. In particular, we were interested in including more stimuli loaded with nasal sounds as well as with a balanced content of oral and nasal sounds. We also decided to direct the investigation to a different language, namely, Brazilian Portuguese. Brazilian Portuguese is characterized by both phonetic and phonological vowel nasalization. While English vowels tend to be nasalized only by assimilation (they are adjacent to a nasal phoneme), Brazilian Portuguese vowels can be nasalized by assimilation in words such as "janela" [3ẽéncle] (window) and "caneta" [kẽe'nete] (pen) as well as in otherwise oral phoneme sequences in words such as "lã" ['lẽe (wool) and "pão" ['pẽw] (bread) [22]. If oral-nasal balance were influenced by voice focus, this effect should be particularly pronounced in Brazilian Portuguese. Based on the findings of the previous study and the model predictions by Rong and Kuehn [19], the first hypothesis was that the forward focus condition would yield higher nasalance scores than the normal speaking condition. The second hypothesis was that the backward focus condition would yield lower nasalance scores than the normal speaking condition.

Apart from the research hypotheses, it was expected, based on normative nasalance values, that nasal stimuli would have higher scores than balanced stimuli, which in turn would have higher scores than oral stimuli [11]. Nasalance scores were expected to be consistent across repetitions.

\section{Methods}

\section{Participants}

Ten female participants with a mean age of 22 years and 6 months (SD 1.38) were recruited from the student population of the Fonoaudiologia program at the Universidade Estadual Paulista "Júlio de Mesquita Filho" in Marília, SP, Brazil. According to their self-report, the speakers had normal hearing, no history of hyper- or hyponasality, and no nasal congestion at the time of data collection. The absence of voice and speech disorders was verified perceptually by 3 experienced speech-language pathologists (V.C.C.M., L.C.B., E.M.G.F.). All participants spoke Brazilian Por- 
Table 1. Mean nasalance scores of 3 repetitions of 9 stimuli in 3 conditions $(n=10)$

\begin{tabular}{lllll}
\hline Stimuli & Average nasalance & \multicolumn{4}{l}{ Nasalance by condition } & \\
\cline { 3 - 5 } & & normal & forward & backward \\
\hline Oral 1 (Dudu) & $17.84(7.51)$ & $16.83(4.92)$ & $21.43(10.03)$ & $14.96(4.75)^{\mathrm{a}}$ \\
Oral 2 (Gostou) & $18.67(9.15)$ & $18.10(6.60)$ & $23.53(11.42)$ & $14.37(6.26)$ \\
Oral 3 (Viu) & $15.54(8.04)$ & $14.33(4.63)$ & $18.93(11.43)$ & $13.37(5.29)$ \\
\hline Balanced 1 (Arruma) & $43.31(8.34)$ & $41.83(5.67)$ & $49.33(8.60)$ & $38.77(6.82)$ \\
Balanced 2 (O cachorro) & $38.62(6.18)$ & $39.07(3.95)$ & $42.03(6.67)$ & $34.77(5.43)$ \\
Balanced 3 (Flavinho) & $35.39(7.08)$ & $32.77(5.21)$ & $40.10(7.61)$ & $33.30(5.87)$ \\
\hline Nasal 1 (Miriam) & $50.29(8.24)$ & $48.77(6.09)$ & $55.50(9.68)$ & $46.60(5.75)$ \\
Nasal 2 (Monica) & $63.98(7.18)$ & $64.70(5.22)$ & $68.10(7.03)$ & $59.13(6.26)$ \\
Nasal 3 (O nene) & $60.60(7.44)$ & $61.83(5.63)$ & $65.47(5.61)$ & $54.50(6.50)$ \\
\hline
\end{tabular}

${ }^{\mathrm{a}} n=9$, in Oral 1 in backward focus.

tuguese with the accent common to Western São Paulo State. The research procedures were reviewed and approved by the Research Ethics Board at the UNESP Marília.

Participant sample size was determined based on previous research [21, 23]. Assuming a mean nasalance score of 49\% (SD 5) for the nasal loaded passage $O$ nenê in the normal speaking condition and a score of $54 \%$ (SD 5) for the forward focus, we determined a minimum sample size of 8 to achieve a power of 0.8 and an alpha of $p=0.05$ (one-sided). Therefore, a group of 10 speakers appeared sufficient for the purposes of the present study.

\section{Participant Training}

All recordings were made by the second, third, and fourth authors. They demonstrated forward focus and backward focus and explained the concept of vocal tract settings. Particular attention was paid to constant speaking pitch in the different conditions. To achieve a forward focus, the participants were instructed to bring their tongue forward, raise their larynx, and narrow the pharynx. The backward focus required the participants to retract their tongue, lower the larynx, and widen the pharynx, which was demonstrated and facilitated with a yawn-sigh $[2,4]$. The participants were provided an opportunity to practice. The data collection began once the second, third, and fourth authors (V.C.C.M., L.C.B., E.M.G.F.) found that the voice focus was being produced correctly.

\section{Stimuli}

The stimuli consisted of 3 sentences without nasal sounds, 3 sentences with a balanced content of oral and nasal sounds, and 3 sentences loaded with nasal consonants (see Appendix). The sentences were shorter versions of stimuli designed for the purpose of clinical nasalance assessment of oral-nasal balance in Brazilian Portuguese. Seven of these sentences were taken from stimuli designed by the second author (V.C.C.M.) [23] and the remaining 2 sentences were from Trindade et al. [24]. The shortened stimuli were used to make the task more manageable for the participants. The minimum length of stimuli for reliable nasalance assessment has been estimated to be 6 syllables [25]. In clinical practice, higher than normative scores for oral stimuli (without nasal sounds) suggests hypernasality, while lower than normative scores for nasal loaded stimuli suggests hyponasality. For nasometric assessment, phonetically balanced stimuli are of limited use. However, they are a good indicator of oral-nasal balance in the speaker's normal dayto-day connected speech. Therefore, balanced stimuli with oral and nasal sounds were also included in the present study.

All 9 stimuli were shown to the participants on a computer screen presented at eye-level for easy reading. The order of the stimuli was randomized, and they were read 3 times for each speaking condition. The participants were asked to read the stimuli in the normal condition first. The order of the two remaining conditions (forward and backward focus) was randomized. If a participant made an error on a stimulus, they were asked to read it again.

\section{Recording Procedures}

All recordings took place in a sound-treated speech laboratory at the UNESP Marília. The participants were seated with their head in an ultrasound transducer stabilizer (Probe Stabilization Headset; Articulate Instruments, Edinburgh, UK). The separation plate of the headset of the Nasometer 6400 (Kay Pentax, Montvale, NJ, USA) was attached to a custom holder and placed on the speaker's prolabium. The nasometer was calibrated according to the manufacturer instructions on each day of recording. The nasalance sound recordings for each condition were saved to hard disk and measured after the session. The mean nasalance scores for the different test items were recorded.

During the recording, the ultrasound probe was held in a constant position under the participant's chin. The video-feed of the participants' midsagittal tongue was recorded using the Advanced Articulate Assistant hardware and software package (Articulate Instruments, Edinburgh, UK). During the live recording, the ultrasound videos served to confirm that the tongue was being held in a protruded (forward focus) or retracted (backward focus) position. For the purposes of the present study, no measurement of the ultrasound recordings was undertaken.

\section{Data Analysis}

Statistical analyses were completed using NCSS version 8.0 (NCSS, Kaysville, UT, USA). The nasalance scores were analyzed 
Fig. 1. Boxplot of nasalance scores for 3 repetitions of 9 stimuli in 3 conditions (normal, forward focus, and backward focus) $(n=10)$.

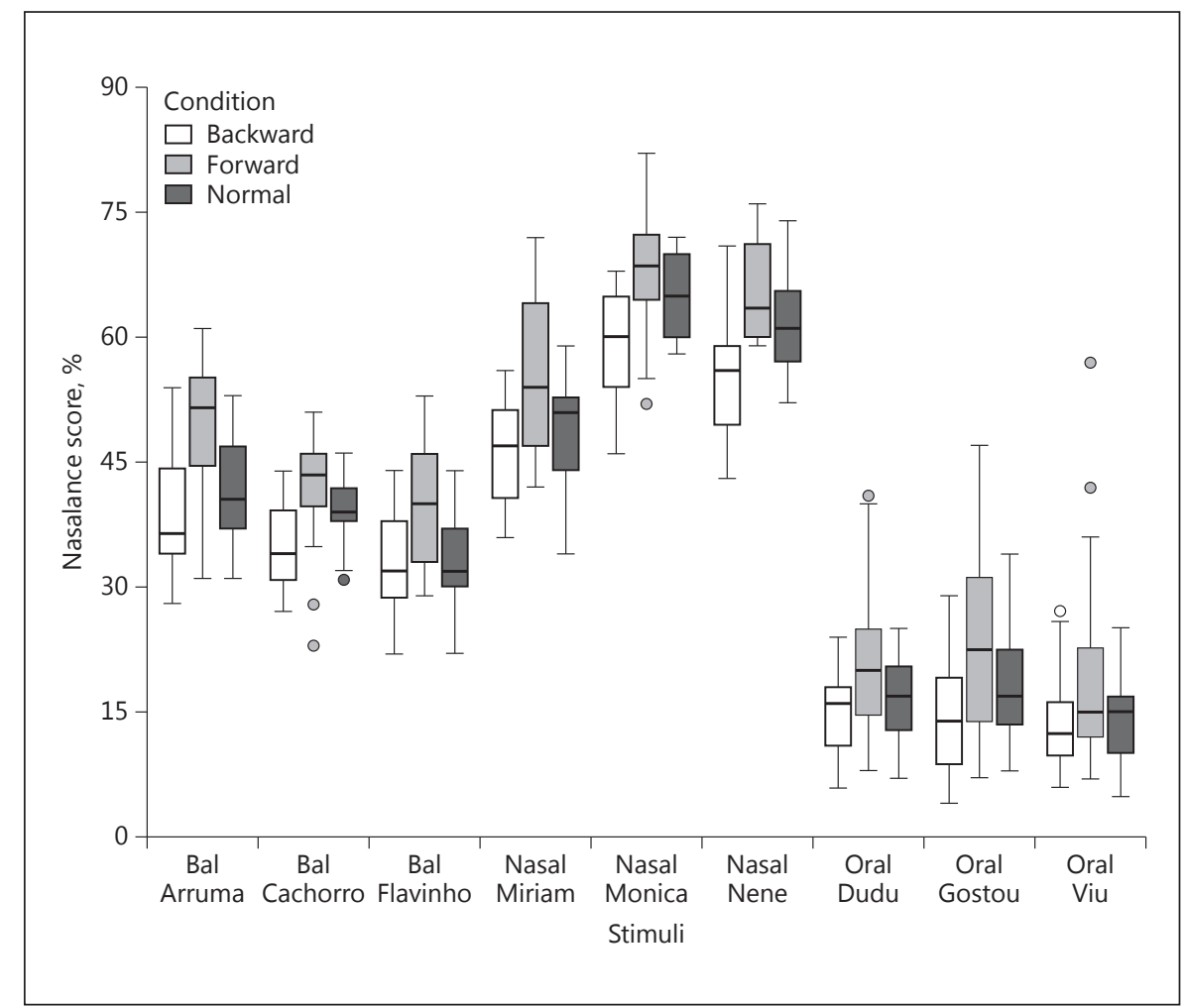

with repeated measures ANOVAs. Where Mauchly's test statistic was significant, the Greenhouse-Geisser adjustment was used. Post hoc testing was carried out with Bonferroni tests. The $p$ value for significance was 0.05 .

\section{Results}

Due to a technical error, the nasalance scores of 1 participant for 1 of the oral sentences $(D u d u)$ were missing. Below, the descriptive results include all available data while the inferential statistics were based on the remaining 8 sentences with complete data for all participants. Table 1 shows the mean nasalance scores for the stimuli by speaking condition as well as averaged across speaking conditions. The box plots in Figure 1 illustrate the findings for the 3 types of stimuli in the 3 speaking conditions. Based on visual inspection, the scores from the forward condition appeared higher and the scores from the backward condition appeared lower than the normal speaking condition.

A three-way repeated measures ANOVA of the nasalance scores was run for 3 repetitions of the 8 stimuli across the 3 speaking conditions. There were significant main effects for stimuli $F(9,63)=240.29, p<0.0001$, and speaking condition $F(2,18)=12.87, p=0.0021$, but not for repetition $F(1,9)=0.48, p=0.6263$. There were no interaction effects.

Across conditions and repetitions, post hoc Bonferroni all-pairwise multiple comparison tests indicated that the stimuli within each oral-nasal balance category (oral, balanced, nasal) were significantly different from the stimuli of the other oral-nasal balance categories. In addition, balanced sentence 3 (Flavinho) was significantly lower than balanced sentence 1 (Arruma), and nasal sentence 1 (Miriam) was significantly lower than both nasal sentence 2 (Monica) and nasal sentence 3 (O nene) (all $p<0.05)$.

Across stimuli and repetitions, the post hoc Bonferroni all-pairwise multiple comparison test found that the backward focus and normal focus conditions generated the lowest mean nasalance scores of $38.85 \%$ (SD 16.85) and $40.18 \%$ (SD 18.02), respectively, while the forward focus had the highest mean nasalance score of $45.38 \%$ (SD 18.90). The backward and normal conditions were both significantly different from the forward condition but not from each other (all $p<0.05$ ). 


\section{Discussion}

The current study expanded a previous investigation of the effect of voice focus on oral-nasal balance [21]. The study was guided by 2 hypotheses. The first hypothesis stated that the forward focus condition would yield higher nasalance scores than the normal speaking condition. This hypothesis was confirmed for all 3 sets of stimuli (oral, balanced, and nasal).

The second hypothesis stated that the backward focus condition would yield lower nasalance scores than the normal speaking condition. This hypothesis was not confirmed. The post hoc tests for the repeated measures ANOVA indicated the difference between the normal and the backward focus speaking conditions but did not reach a $p$ value of 0.05 . However, the mean nasalance values of the stimuli for the backward focus speaking condition were numerically lower. To determine the $p$ value of the difference between the normal and backward focus speaking conditions, a separate $t$ test was run. It suggested a trend towards significance $(p<0.07)$. It is possible that this difference would have been significant in a larger group of speakers.

As expected, there was a stimulus effect whereby nasal stimuli had higher scores than balanced stimuli, which in turn had higher scores than oral stimuli. Marino et al. [23] provided normative nasalance scores by age and gender. The data from the current study could therefore be compared to the normative data for young adult females. In the normal condition, the mean nasalance scores for the oral stimuli ranged from $14.33 \%$ (SD 4.63) to $18.10 \%$ (SD 6.60). This was slightly higher than the normative score of $14.04 \%$ (SD 3.82) reported for a comparable oral passage [23]. The mean scores for the balanced oral-nasal stimuli ranged from $32.77 \%$ (SD 5.21) to $41.83 \%$ (SD 5.67) which was greater than the reported normative mean of $26.90 \%$ (SD 4.00) for a balanced oral-nasal stimulus [23]. The nasal stimuli means ranged from $48.77 \%$ (SD 6.09) to $64.70 \%$ (SD 5.22), which were higher than the normative mean nasalance score of $49.37 \%$ (SD 4.69) reported for a nasal stimulus. The differences can possibly be attributed in part to differences in the sentence stimuli, which were used in shortened versions in the present study. It has also been noted that the nasalance group means show some intrinsic variation between studies and even within speakers $[26,27]$. While the nasalance values measured in the present study appeared higher than the suggested norms, it is unlikely that this difference would have affected the quality of the results. Within the repeated measures design, the participants' nasalance values in the normal speaking condition served as their own control condition for the comparison with the forward and backward speaking conditions. The results of the ANOVA suggested that the changes in nasalance based on the speaking condition were consistent across speakers.

The participants of this study and those of the previous pilot study were all female. Although males have larger vocal tracts than females, the differences in nasalance scores are not always significant [21, 24, 27-30]. When significant differences are found by gender, they tend to be small and not clinically meaningful [23, 32-36]. Nevertheless, more research will be needed to confirm the observed effect for male speakers.

The results expanded the previous findings from a group of Canadian English speakers [21] to participants speaking Brazilian Portuguese. An important improvement of the current study was the inclusion of speech stimuli with a balanced phonetic content. Nasalance testing of clinical speakers usually focuses on oral and nasally loaded stimuli, which represent phonetic extremes. However, normal connected speech has a more balanced phonetic content. Therefore, the oral-nasal balance requirements of conversational speech are more accurately represented by the balanced stimuli.

In the previous study [21], the success of the speakers in moving their speaking focus forward or backward was analyzed using long-term average spectra (LTAS), which demonstrated an upward or downward movement, respectively, of the first spectral peak, similar to the spectral changes described by Sundberg and Nordström [3] for a single trained singer. In the present study, the speakers' productions were not analyzed with LTAS. The participants all had training in phonetics and vocal tract anatomy. In addition, their productions were verified perceptually by the 3 experienced speech-language pathologists carrying out the experiment. Since the changes in nasalance values were consistent across the participants for the different speaking conditions, no additional external validation was undertaken. In future research, it would be interesting to investigate the acoustic consequences of changes of the speaking focus in more detail.

These limitations notwithstanding, the present study successfully corroborated the findings from the pilot study [21]. The findings confirmed that voice focus can affect oral-nasal balance, especially in speech stimuli that are balanced or loaded with nasal sounds. The effect has now been shown in 2 languages, 1 with and 1 without phonological vowel nasalization. Future studies are needed to explore the effect of voice focus in other languages, as well as to verify that a similar effect is observed in male 
speakers. In a next step, it will be of interest to explore the extent to which speaking focus adjustments could be used to improve the oral-nasal balance of hypernasal speakers with cleft palate. Based on the findings from this study as well as the previous study [21] and a computer model [19], it would be expected that the backward speaking focus should reduce nasalance scores and perceived hypernasality. It remains to be seen whether it is possible for hypernasal speakers with cleft palate to use speaking focus adjustments to achieve changes in oral-nasal balance that would be measurable and perceptually relevant.

\section{Appendix}

Speech stimuli based on Marino et al. [23] and Trindade et al. [24] (indicated with *).

Oral

Dudu visitou o bosque.

Viu o pulo do sapo.

Gostou do peixe.

\section{Balanced}

O cachorro do Nino.

Arruma seu bercinho.

Flavinho chamou o João.*

Nasal

Monica mima o nenê.

Miriam lambeu o limão.*

O nene mama.

\section{Acknowledgements}

The study was supported by the São Paulo Research Foundation (FAPESP, grants No. 2012/23899-6 and 2016/01583-8). The authors wish to thank the participants of this study.

\section{Disclosure Statement}

The authors have no conflicts of interest to disclose.

\section{References}

1 Boone DR: Is Your Voice Telling on You? ed 2. San Diego, Singular, 1997.

2 Boone DR, McFarlane SC, Von Berg SL, Zraick RI: The Voice and Voice Therapy, ed 8. New York, Allyn \& Bacon, 2010.

3 Sundberg J, Nordström P-E: Raised and lowered larynx - the effect on vowel formant frequencies. Speech Transmission Laboratory Quarterly Progress and Status Report. Stockholm, Royal Institute of Technology, vol 2-3, pp 35-39.

4 Boone DR, McFarlane SC: The Voice and Voice Therapy, ed 6. Boston, Allyn and Bacon, 2000.

5 Fritzell B: The velopharyngeal muscles in speech: an electromyographic and cineradiographic study. Acta Otolaryngol 1969; (suppl)250:1-81

6 Moon J, Smith A, Folkins J, Lemke J, Gartlan $\mathrm{M}$ : Coordination of velopharyngeal muscle activity during positioning of the soft palate. Cleft Palate Craniofac J 1994;31:45-55.

7 Moll KL: A cinefluorographic study of velopharyngeal function in normals during various activities. Cleft Palate J 1965;2:112-122.

8 Flowers CR, Morris HL: Oral-pharyngeal movements during swallowing and speech. Cleft Palate J 1973;10:181-191.

9 Shprintzen RJ, McCall GN, Skolnick ML, Lencione RM: Selective movement of the lateral aspects of the pharyngeal walls during velopharyngeal closure for speech, blowing, and whilstling in normals. Cleft Palate J 1975;12. 51-58.

10 Lewis KE, Watterson T, Quint T: The effect of vowels on nasalance scores. Cleft Palate Craniofac J 2000;37:584-589.

11 Kummer AW: Simplified Nasometric Assessment Procedures (SNAP): Nasometer TestRevised. Lincoln Park, Kay Elemetrics, 2005.

12 Gildersleeve-Neumann CE, Dalston RM: Nasalance scores in noncleft individuals: why not zero? Cleft Palate Craniofac J 2001;38:106-111.

13 Awan SN, Omlar K, Watts CR: Effects of computer system and vowel loading on measures of nasalance. J Speech Lang Hear Res 2011;54: 1284-1294.

14 Croft CB, Shprintzen RJ, Rakoff RJ: Patterns of velopharyngeal valving in normal and cleft palate subjects: a multiview videofluoroscopic and nasendoscopic study. Laryngoscope 1981;91:265-271.

15 Kummer A: Cleft Palate and Craniofacial Anomalies - Effects on Speech and Resonance, ed 2, New York, Delmar Cengage Learning, 2008.

16 Hixon TJ, Weismer G, Hoit JD: Preclinical Speech Science: Anatomy Physiology Acoustics Perception. San Diego, Plural Publishing, 2008.

17 Sweeney T: Nasality - assessment and intervention; in Howard S, Lohmander A, (eds): Cleft Palate Speech: Assessment and Intervention. Hoboken, Wiley, 2012, pp 199-220.
18 Kuehn DP, Moon JB: Velopharyngeal closure force and levator veli palatini activation levels in varying phonetic contexts. J Speech Lang Hear Res 1998;41:51-62.

19 Rong P, Kuehn D: The effect of articulatory adjustment on reducing hypernasality. Speech Lang Hear Res 2012;55:1438-1448.

20 Bressmann T, Anderson JD, Carmichael RP, Mellies C: Prosthodontic management of hypernasality: two very different cases. Can J Speech-Lang Path Audiol 2012;36:50-57.

21 De Boer G, Bressmann T: Influence of voice focus on oral-nasal balance in speech. J Voice 2015, Epub ahead of print. http://www. sciencedirect.com/science/article/pii/ S089219971500199X.

22 Silva TC: Fonética e fonologia do português: roteiro de estudos e guia de exercícios, ed 9. São Paulo, Contexto, 2007.

23 Marino VCC, Dutka JCR, de Boer G, Cardoso VM, Ramos RG, Bressmann T: Normative nasalance scores for Brazilian Portuguese using new speech stimuli. Folia Phoniatr Logop 2016;67:238-244.

24 Trindade IEK, Genaro KF, Dalston RM: Nasalance scores of normal Brazilian Portuguese speakers. Braz J Dysmorphol Speech Hear Disord 1997;1:23-34.

25 Watterson T, Lewis KE, Foley-Homan N: Effect of stimulus length on nasalance scores. Cleft Palate Craniofac J 1999;36: 243-247. 
26 De Boer G, Bressmann T: Comparison of nasalance scores obtained with the Nasometers 6,200 and 6,450. Cleft Palate Craniofac J 2014; 51:90-97.

27 De Boer G, Bressmann T: Application of linear discriminant analysis to the nasometric assessment of resonance disorders: a pilot study. Cleft Palate Craniofac J 2015;52:173182.

28 Prathanee B, Thanaviratananich S, Pongjunyakul A, Rengpatanakij K: Nasalance scores for speech in normal Thai children. Scand J Plast Reconstr Surg Hand Surg 2003;37:351355.
29 Sweeney T, Sell D, O’Regan M: Nasalance scores for normal Irish-speaking children. Cleft Palate Craniofac J 2004;41:168-174.

30 Hirschberg J, et al: Adaptation of nasometry to Hungarian language and experiences with its clinical application. Int J Pediatr Otorhinolaryngol 2006;70:785-798.

31 Lee A, Browne U: Nasalance scores for normal Irish-English speaking adults: a crossgender comparative study. Logoped Phoniatr Vocol 2013;38:167-172.

32 Brunnegard K, Van Doorn J: Normative data on nasalance scores for Swedish as measured on the Nasometer: influence of dialect, gender, and age. Clin Linguist Phon 2009;23:5869.
33 Seaver EJ, Dalston RM, Leeper HA, Adams LE: A study of nasometric values for normal nasal resonance. J Speech Hear Res 1991;30: 522-529.

34 Van Lierde KM, Wuyts FL, De Bodt M, Van Cauwenberge P: Nasometric values for normal nasal resonance in the speech of Flemish adults. Cleft Palate Craniofac J 2001;38:112118.

35 Mishima K, Sugii A, Yamada T, Imura H, Sugahara T: Dialectal and gender differences in nasalance scores in a Japanese population. J Craniomaxillofac Surg 2008;36:8-10.

36 Mayo CM, Mayo R: Normative nasalance values across languages. ECHO 2011;6:22-32. 